\title{
AN INNOVATIVE IDEA TO DISCOVER THE TREND ON MULTI- DIMENSIONAL SPATIO-TEMPORAL DATASETS
}

\author{
N. Naga Saranya ${ }^{1}$, M. Hemalatha ${ }^{2}$ \\ ${ }^{1}$ Assistant Professor, Department of Computer Applications, Tamil Nadu, India \\ ${ }^{2}$ Professor, Department of Computer Science, Tamil Nadu, India
}

\begin{abstract}
Spatio-temporal data is any information regarding space and time. It is frequently updated data with 1TB/hr, are greatly challenging our ability to digest the data. Thereupon, it is unable to gain exact information from that data. So this research offers an inno vative idea to discover the trend on multi-dimensional spatio-temporal datasets. Here it briefly describes the scope and relevancy of spatiotemporal data. From that, gain the depth knowledge of spatio-temporal recent research process to discover the trend.
\end{abstract}

Keywords: Spatio-temporal Data, Applications of Spatio-temporal data, Problem Definition, Contributions

\section{INTRODUCTION}

Data mining is the analysis of observational data sets to find unsuspected relationships and to summarize the data in novel ways that are both understandable and useful to the data owner. Extracting interesting and useful patterns from spatio temporal database is more difficult than extracting the corresponding patterns from traditional (fixed) numeric and categorical (clear) data due to the complexity of spatio temporal data types, spatio - temporal relationships, and spatio - temporal autocorrelation. Spatio-temporal data is any information relating space and time.

Geospatial data is regularly updated data with $1 \mathrm{~TB} / \mathrm{hr}$, are greatly challenging our ability to digest the data. With that data, it is unable to gain exact information. Here the data may lose. Geospatial data take a view of geospatial phenomena, here it captures the spatiality. If it evolves over time, it captures the spatiality as well as the temporality. From that we are able to know the process and events of spatio-temporal data. Knowledge of extracting spatio-temporal data gives better prediction of its process and events.

Finding the trend is most important task in spatio-temporal data. Particularly it is to analyze trajectories movement, animal movements, mating behavior, harvesting, soil quality changes, earthquake histories, volcanic activities and prediction etc. Spatial, temporal and/or spatio-temporal data mining looks for patterns in data using the spatio-temporal attributes. Trend discovery cannot be directly performed well. The proposed research performs trend discovery by combining the principles of clustering, association rule mining, generalization and characterization.
The significance of spatio-temporal data is continuously updated data with $1 \mathrm{~TB} / \mathrm{hr}$. It is terribly massive and hugedimensional geographic and spatio-temporal datasets.

Meteorology
Biology
Crop sciences
Forestry
Medicine
Geophysics
Ecology
Transportation, etc

And we can apply this proposed algorithm to any kind of data mining applications. Especially when we apply to spatiotemporal databases, it gives most significant results. When trend discover algorithm is used, the rate of the retrieval is fast and results obtained were accurate.

The objective of this research is to develop a novel algorithm for trend discovery on multi dimensional Spatio-temporal databases. Objectives are,

Predict the knowledge

Retrieve hidden information

Discover Trend

Future Usage

\section{LITERATURE REVIEW}

\subsection{Clustering in Spatio-Temporal Data}

Literature survey have been made on of cluster-ing the moving data objects in the trajectory dataset the basis of trajectories representation, moving object point, similarity, clustering algorithms. Data can be viewed as three forms like spatial aspects, temporal aspects and spatio-temporal aspects. The problem of clustering of moving objects in spatial networks 
environment is ad-dressed (Jidong Chen et al., 2007). Since the clustering process is seems to be difficult the author proposed a framework that are divided into two sections. It can be done through performing various methods on CBs for constant maintaining and periodical construction of clusters. Results show that the proposed framework outperforms inroad network. The author deploys multi-scale wavelet transforms and self-organizing map with neural networks to mine air pollutant data (Sheng-Tun Li and Shih-Wei Chou, 2000). Significance of using Wavelet transform method is to generate data and time variant features for detecting the air pollutant spatial data in a specific time variant. This wavelet transform method greatly reduces the local small regions using small scale input data and improve the over-smoothed regions using one large scale input data.

Moving objects in trajectory database can be identified with the help of free moving trajectory context and constrained trajectories (Ahmed Kharrat et al., 2008). Spatial shapes are identified to evaluate the OWD (One Way Distance) in both continuous and discrete cases and trajectories similarity measures are later dis-cussed (Lin et al., 2005).

The problem of moving object with two datasets with the help of time series algorithm is investigated (Hoda M. O. Mokhtar et al., 2011). And both simulated and real time data has been used for the study. A new similarity measures based on huge dimensional mobile trajectories is presented (Sigal Elnekave et al., 2007). The uniform geometric model has been proposed (O. Gervasi et al., 2005) for clustering and query timedependent data. Three dimensional visualization tools to help the user visualization and time taken for query clustering that are dynamic in nature.

A study on historical trajectories of moving ob-jects by proposing an algorithm to discover moving clus-ters are performed (Kalnis et al., 2005). Sequence of spatial clusters that appear in consecutive snapshots of the object movements is termed as moving clusters and other sequence of clusters tends to share the most common objects. That can be detected using clusters at consecutive snapshots with high time complexity. The problem of finding clusters both in dynamic and continuous nature within 2D Euclidean space is (Christian S. Jensen et al., 2007) analyzed. Using this concept the user can easily generate cluster necessary features within reduced time constraints. The insertion and deletion of clustering scheme has not done efficiently. An experimental result shows that the proposed method outperforms when compared to other existing methods. Clusters for mobile object trajectories to find the movement patterns based on cluster centroids are (Sigal Elnekave et al., 2007) evaluated.

To predict the object location, the movement pattern is used. Precision and recall values are used to measure the performance of clusters. Threshold values are kept to remove the exceptional data points. Several existing clustering methods have been done to calculate the overall partition of data sets so as to provide some meaningful information about clusters. But it may not applicable for moving object databases with huge dimensional spatio-temporal data sets. An interesting approach called model based concept clustering (MCC) to identify the moving object in video surveillance is (Jeongkyu Lee et al., 2007) provided. It includes three steps namely model formation, model-based concept analysis and concept graph generation. The results show that the MCC works well in terms of quality when compared to other existing methods.

The goal of trajectory clustering is to find simi-lar movement traces. Many clustering methods have been proposed using different distance measures between trajectories. The similar portions of sub trajectories are (Lee. J.G et al., 2007) discovered. Note that a trajectory may have a long and complicated path. Since the two trajectories are similar in some sub-trajectories, they may not be similar as a whole. Similar sub-trajectories are useful, when one considers regions of special interest in analysis part. This observation leads to the development of new sub-trajectory clustering algorithm named as TRACLUS (Lee. J.G et al., 2007). This method discovers clusters by grouping sub-trajectories based on density. Whereas each trajectory must be partitioned into line segments using Minimum Description Length (MDL). Then, density-based clustering is applied on the segments to represent the sub-trajectories that are summarized over all the line segments in the cluster.

The predestination method to know the history of a driver's destination, along with its behaviors, to pre-dict a trip progress of a driver is (Krumm.J and E.Horvitz, 2006) described. The driving behaviors pos-sess the starting point of drier, its destinations and also its efficiency to estimate the trip times and position of the truck. Four different probabilistic aspects and ma-thematical principles to create a probability grid of expected destinations. The authors introduce an open-world model of destinations that helps the algorithm to work well with small of training data at the beginning of the training period by accounting the behavior of users (I.e.what they visited previously) and unobserved loca-tions based on trends in the data and the background properties related to the concerned locations. The 3,667 different driving trips show the error for two kilometers at the trip's halfway point. However this technique predicts the driver's destination, at any given point of time. In case of linear functions the object movement may exhibit different movement patterns.

A trajectories cluster contains similar periodic trajectories. Trajectories in the same cluster contain as much similar MBBs that are close to space and time. The centroid of a cluster in a trajectory databases groups similar trajectory to represent a movement pattern of a given object. Since the genetic clustering algorithm handles input of trajectory data to bound intervals (trajectories) in spite of numeric vectors Y X T hours 
are estimated. The K-Means algorithm for clustering trajectories based upon the amount of data and its corresponding similarity measures in a spatial temporal version is also (Elnekave S et al., 2007) used. It makes use of new centroid structure and updating me-thod respectively. It adapt the incremental clustering approach to promote the clustering of the first training data window (e.g. trajectories during the first month of data collection), when no pasts data are available and clustering for the corresponding subsequent windows, by utilizing clustering centroids and thereby improving the performance of various sequences of incremental clustering processes (Elnekave $S$ et al., 2007). Limited updation are needed to pursue the movement behavior of the same object which stays relatively stable.

More recently (Spiliopoulou et al., 2006), a framework called MONIC has been proposed to model the traces of cluster transitions. Specifically, the data can be clustered at varying timestamps using bisecting K-means algorithm to detect the changes of clusters at various timestamps. Unlike the above two works, which analyze the relations between clusters after the clusters are obtained, here author aims to predict the possible cluster evolution to guide the clustering. Finally, we note that clustering of moving objects involves future-position modeling. In addition to the linear function model, which has been involved in most work, a recent proposal considers nonlinear object movement (Tao.Y et al., 2004).

The key aspect is to obtain a recursive motion function to predict the future positions of a moving ob-ject depending upon the positions in the recent past. However, this approach is more complex than the widely adopted linear model and complicates the analysis of several interesting spatio-temporal problems. Thus, we use the linear model. But it is not feasible to find work on clustering in the literature devoted to kinetic data structures (Basch.J et al., 1999).

Recently, a new framework has been proposed for segmenting trajectories for enabling line segments (Lee.J.G et al., 2008, and Lee.J.G et al., 2007). These line segments are grouped together to build the clusters. However, time has not been considered in this system (Lee.J.G et al., 2008, and Lee.J.G et al., 2007), which makes some line segments to be clustered together even though they are not "close" when time is considered. Nevertheless, such approaches for clustering moving objects cannot solve the flock pattern query since: (1) they use different criteria when joining the moving ob-ject clusters for two consecutive time instances; (2) they employ clustering algorithms, and therefore no strong relationship between all elements are enforced; (3) mov-ing clustering does not require the same set of moving objects to stay in a cluster all the time for the specified minimum duration.

The distance between two trajectory segmenta-tions at time $\mathrm{t}$ as the distance between the rectangles at time $t$, and the distance between two segmentations is the sum of the distances between them at every time instant is (Anagnostopoulos et al. (2006) defined. The distance between the trajectory MBRs is a lower bound of the original distance between the raw data, which is an essential property for generating the correctness of results for most mining tasks. The similarity of trajectories along time is computed by analyzing the way the distance between the varies trajectories (D'Auria, et al., 2005). More precisely, for each time instant the positions of moving objects is compared at that moment, therby aggregating the set of distance values.

The generic definition of clustering is usually redefined depending on the type of data to be clustered and the clustering objective. Different and scalable clus-tering algorithms have been proposed (Iwerks Glenn S et al., 2003, and LiuWenting et al., 2008). The authors pro-pose a clustering technique (Zhang Qing and Lin Xu-emin, 2004), that uses a distance function which com-bines both position and velocity differences, they employ the k-center clustering algorithm (Gonzalez, 1985) for histogram construction. The authors represent a trajectory as a list of (MBB) minimal bounding boxes (Sigal Elnekave et al., 2008).

Trajectory clustering problem is further investi-gated (Dino Pedreschi Margherita D'Auria and Mirco Nanni, 2004). In this work, a clustering technique called temporal focusing was introduced to make use of the intrinsic semantics of the temporal dimension and therby promoting the quality of trajectory clusters. Based on this dependency, the densitybased clustering algorithm has been proposed to trajectory data for estimating a notion of distance between trajectories. Clustering of moving objects to optimize the continuous spatio-temporal query execution has been (Rimma V. Nehme and Elke A. Rundensteiner, 2006) used. The moving cluster can be represented by a circle. And a unified framework for Clustering Moving Objects in spatial Networks (CMON) is (Chen Jidong et al., 2007) proposed. In this architecture the clustering process is divided into the continuous maintenance of cluster blocks $(\mathrm{CBs})$ and the periodical construction of clusters with different criteria based on CBs. A CB groups a set of objects on a road segment in close proximity to each other at present and in future.

The moving clusters from historical trajectories of objects is (In Panos Kalnis et al., 2005) discovered. Important events like collision among micro-clusters are also identified. Based on the historical trajectories of moving objects a new algorithm has been proposed. A moving cluster is defined as a sequence of spatial clusters that appear in consecutive snapshots of the object movements and subsequent spatial clusters share a large number of common objects. A clustering technique (Hoda M. O. Mokhtar et al., 2011), that is close to the partition and group framework proposed (Lee Jae-Gil et al., 2007) to partition a trajectory into a set of line segments and then groups similar line segments together into a cluster. Clustering approaches to enable the common aspects of sub- 
trajectories from a trajectory database has benn (Hoda M. O. Mokhtar et al., 2011) proposed. This aspect makes the approaches more accurate than techniques that cluster based on the whole trajectory behavior. The proposed algorithms uses the idea of recapping to create a list of clusters that are visited by different trajectory's segments developed by an approximate clustering algorithm using the visited cluster list so as to predict the future motion pattern in the trajectory databases.

\subsection{Outlier Detection in Spatio-Temporal Data}

Data objects that appear away from particular consistency in database are called outliers (Barnett and Lewis, 1994). Usually uncommon and remarkable ac-tions of data will be considered as anomalies or noise. Outlier detection is classified as distribution-based, depth-based and distance based methods. First, the dis-tribution-based approach uses standard statistical distri-bution, secondly, the depth-based technique divides the data objects into an $\mathrm{n}$ number of dimensional space (i.e., $\mathrm{n}$ is the total number of attribute in that space) and finally the distance-based approach estimates the amount of database objects in a specified distance from a target object (Ng. R.T, 2001). The non-spatial referenced objects vary from the other objects by means of spatial reference of its neighborhood called spatial outliers. All spatially referenced objects may not differ from the entire objects only some of its specified location differs from the other in nature (Shekhar et al, 2003).

Distribution based approach have been used extensively in case of analyzing spatial outliers (Shekhar et al, 2003). It includes two types of outlier detection methods namely single and multidimensional outlier detection method.

The problem of spatial-temporal outlier (STO) in which the spatio - temporal objects differs greatly from both spatial and temporal objects in terms of rela-tionship are addressed (Tao Cheng et al., 2004). To im-prove the performance of both semantic aspects and dynamic aspects of spatio-temporal data in multi-scales environment, the STO is proposed.

Trajectory outliers are of two kinds. First, a moving object can be an outlier in terms of its neighborhood or it does not follow the similar paths. It can be detected by calculating the distance between two fixed moving objects. Secondly, the outlying sub-trajectory may not follow the common aspects among other sub trajectories respectively. This method includes many challenging issues and can be solved by partition-and-detect framework (Lee. J.G et al., 2008).

\subsection{Feature, Locations, Patterns from Spatio- temporal Data}

The main motivation of association rule is to find the regularities between the items and their support and confidence value in huge volume of data sets (Diansheng Guo and Jeremy Mennis, 2009). Finding the rule generation using association rule mining, sometimes it may seriously affects the threshold; support and confidence value (R.J. Kuoa et al., 2011). To overcome this drawback, a new framework has been proposed for assigning the threshold automatically by the system with the help of association rule mining technique and the working efficiency is more.

Normal association rule generation steps for data set are same to generate the rule in spatio-temporal database also. It considers the spatio-temporal predicates and properties. Number of authors has been discussed about rule mining in spatio-temporal data (Appice et al., 2003, and Han et al., 2001, and Koperski et al., 1995, and Mennis et al., 2005).

But for finding the spatio-temporal data set feature, locations, patterns from the co-location point etc, are very difficult for generating the rule using association rule mining method (Shekhar and Huang, 2001). Features of those are frequently located together, such as a certain species of bird tend to habitat with a certain type of trees. And for finding the spatiotemporal patterns and their co-locations, and the measuring process, a new algorithm has been proposed (Huang et al., 2006, and Lu et al., 2008 and Shekhar et al., 2008). Finally the features are extracted from the spatio-temporal data sets and discover the trend using association rule mining (Zhang Xuewu , 2008).

Particle swarm optimization (PSO) first searches the best fitness for each and every particle with the help of minimum support and confidence value denoted as threshold (Manisha Gupta, 2011). Based on the literature study, the author concluded that the PSO will suggest the suitable threshold for the searching the particle. Spatio-temporal auto correlation and regression using algebra formula is also done with the help of spatio-temporal association rule mining (Corbett. J, 1985). Here spatio-temporal data structure and their auto correlations are calculated by algebra formula.

The measurement of auto correlation, frequent item set of autocorrelation using algebra formula has been done with the help of spatio-temporal data structure such as points, lines, curves, regions, etc (Jiangping Chen, 2008). Several author discussed the role of association rule mining in spatial mining, temporal mining, privacy preservation mining, data stream mining, utility mining, and etc using optimization techniques (Maragatham. G, 2012). This survey guides the upcoming researchers to know the growth of association rule mining role in different types of mining.

By applying association rule in spatio-temporal or non spatiotemporal data, a specific format data has been delivered (Imam Mukhlash and Benhard Sitohang, 2007). Chaotic particle swarm optimization (CPSO) method with an acceleration strategy has been proposed for finding the optimization in spatio-temporal data (Cheng-Hong Yang, 2009). Acceleration strategy combined with chaotic particle 
swarm optimization (CPSO) becomes Acceleration chaotic particle swarm optimization (ACPSO). ACPSO effectively find out the cluster centers and the global fitness for the certain particle. But this system cannot able to work properly to find out the best particle in huge volume of data.

The combination of PSO and BP based neural network system is developed to train the network. Here it produces the shortest time, high accuracy with precision and recall rate, fast disappearance, etc (ZhongLiang Fu and Bin Wan, 2009). Generating the rule and finding the cluster center using optimization techniques also discussed by number of authors (Samadzadegan. F and S. Saeedi, 2009). Here the optimization method (PSO) reduces the time consumption and produce the better performance, while finding the cluster centers and searching the best fitness of each particle. But when the concentration of local and global best particle, it may miss to work properly.

A new framework has been proposed for finding the best fitness in terms of comprehensibility and accuracy (Veenu Mangat, 2010) using association rule mining technique. This system reduces the time and space complexity and it finds the optimal value for the variables in the optimization algorithm. The features of frequently co-located patterns are discovered for the spatio-temporal Boolean features. The problem of spatio-temporal co-location pattern is difficult, when applying spatial association rule. Because there is no normal notion of transactions which are embedded in continues geographic space.

\section{DRAWBACKS OF EXISTING SYSTEM}

Clustering of spatio - temporal data is a difficult prob-lem that is compared to various fields and applications.

o The Major and common drawbacks are high dimensionality of data (2D or 3D), initial error propaga-tion, high dimensional data complexity.

o Clustering of space and time related data, spa-tiotemporal clustering methods focus on the specific characteristics of distributions in 2-D or 3-D space, while general-purpose high-dimensional clustering method have limited power in recognizing spatiotemporal pat-terns that involve neighbors.

o In human computer interaction, the clustering techniques may not work properly to find out the complex patterns in huge volume of spatio-temporal data sets.

Outlier Detection - Spatio-temporal neighbors which is occurs in spatio-temporal outliers are very conflict, even the non spatio-temporal values are normal for the rest of the objects of the same class.

o Spatio-temporal outlier detection may not prop-erly work with non-spatial or non-temporal datasets. Even if the non-spatial attributes are separated, it cannot able to predict the outliers from spatio-temporal datasets.

Rule mining in spatio-temporal data - Dependency Anal-ysis existing algorithms drawbacks are, while incorporat-ing local search in high-dimensional spatio-temporal data, the performance is very low and it faces the diversity problem. In the optimization process, the existing algorithms are not able to work with partial optimism.

o In that stage, the algorithm will easily affect the speed and direction of the particle and it may not work properly to do their remaining iterations.

o And the existing algorithms cannot able to solve the issues of huge dimensional, unsystematic datasets in the stage of particle search and moving object search.

Trend discovery in spatio-temporal data- existing algorithms drawbacks, while discovering the trend in spatiotemporal data are as follow,

o The anomalies will occur in the existing system, while it concentrates the graph alteration. Here the in-formation may loss badly. So the necessities of the table scan may increase more.

o In generalization process face problem to identify the description of data while doing the path relation and their hierarchy.

o Spatio-temporal dependency process faces sev-eral problems while finding the dependency of large spa-tiotemporal data.

To overcome these drawbacks, we propose new algo-rithms for discovering the best trend in spatio-temporal data sets.

\section{PROBLEM DEFINITION}

The performance of trend discovery analysis is hindered in spatio-temporal data due to the following reason:

- Clustering of massive spatio-temporal data is cumbersome. Features of the data are often preselected, yet the properties of different features and feature combinations are not well investigated in the huge spatio-temporal data sets. Finding appropriate features to form a cluster group is essential for better search.

- Cluster accuracy is less, so it requires deviation and outlier detection analysis for high dimensional spatiotemporal clustered data.

- Rule generation accuracy of spatio-temporal data is less, while it predicts the value of one attribute with the help of another attribute value over a period of time. So it requires optimization process for rule generation.

- To know about the compact description of data, it needs better generalization and characterization method.

- Trend discovery of spatio-temporal data accuracy is very less, while it's going for the huge dimensional data sets. 


\section{CONTRIBUTIONS OF THIS RESEARCH}

\section{WORK}

Phase 1 gives the solutions to the challenges in segmenting the spatio - temporal data by Proposed PANN algorithm

Phase 2 removes the deviations and outliers of spatio temporal data accurately by improved STOD Technique

Phase 3 generates the rules for dependency analysis of spatio temporal data by proposed IESO technique

Phase 4 generalize and characterize the spatio - temporal data by improved GAOI technique

Phase 5 Finally the Proposed GPLDE algorithm is developed for the further trend discovery of spatio - temporal data.

Fig 1 shows that the entire framework of proposed research.

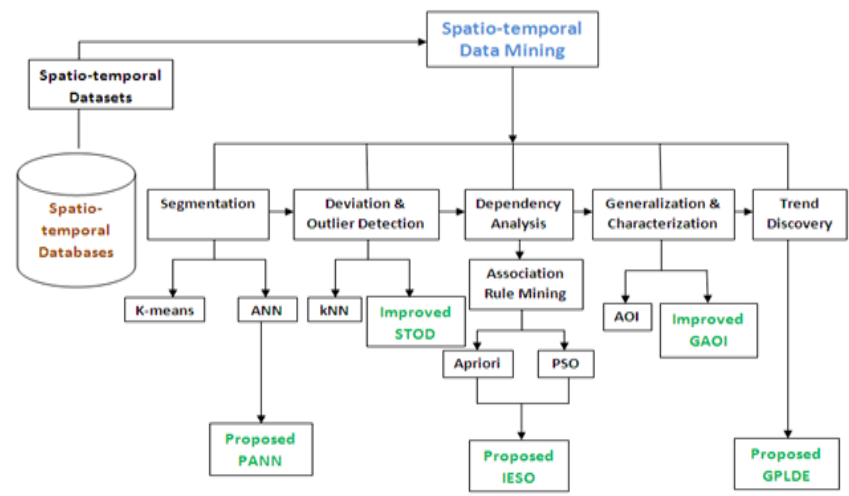

ANN-Artificial Neural Network PANN-Principle Artificial Neural Network OD-Outlier Detection STOD - Spatio-temporal Outlier Detection PSO - Particle Swarm Optimization IESO - Intelligent Swarm Ant Optimization AOI-Attribute Oriented Induction GAOI-Generalized Attribute Oriented Induction GPLDE - Gaussian Pairwise Linear Difference Estimator

Fig 1 Entire Framework of Proposed Research

\section{CONCLUSIONS}

This research offers an innovative idea to discover the trend on multi-dimensional spatio-temporal datasets. Here it briefly describes the scope and relevancy of spatio-temporal data. From the literature survey it has listed a number of issues. And also it contributes several phases, in which each and every phase output must be very helpful to go for the next phase input. From that, gain the depth knowledge of spatio-temporal recent research process to discover the trend. The proposed algorithms evaluation process of this research will be learned in our next research paper.

\section{REFERENCES}

[1] Adam, N.R., V.P. Janeja and V. Atluri, 2004. Neighbourhood based detection of anomalies inhigh dimension spatio-temporal sensor datasets. ACM symposium on Applied Computing, Nicosia, Cyprus., 1: $576-583$.

[2] Ai, C. and X. Chen, 2003. Efficient estimation of models with conditional moment restrictions containing un-known functions. Econometrica., 7: 1795-1843.
[3] Anagnostopoulos. A., M. Vlachos., M. Hadjieleftheriou., E Keogh and P.s. Yu, 2006. Global Distance-Based Segmentation of Trajectories. KDD’06, Philadelphia, Pennsylvania, USA.

[4] Anselin, L. and A.K. Bera, A.K, 2002. Spatial dependence in linear regression models with an introduction to spatial econometrics. In: Ullah, A., Giles, D.E.A. (Eds.), Handbook of Applied Economics Statistics. Marcel Dekker, New York.

[5] Auroop. RR., Ganguly and Karsten Steinhaeuser, 2008. Data Mining for Climate Change and Impacts. IEEE International conference on Data mining workshop, ICDMW., 385-394.

[6] Basile, R. and B. Gress, 2004. Semi-parametric spatial auto-covariance models of regional growth behavior in Europe. Mimeo. Dept. of Economics, UC, River-side.

[7] Benkert. M., J. Gudmundsson., F. H“ubner and T. Wolle, 2008. Reporting flock patterns. Comput. Geom. Theory Appl., 41(3):111-125.

[8] Cheng-Hong Yang et al,. 2009. Accelerated Chaotic Par-ticle Swarm Optimization for Data Clustering. Inter-national Conference on Machine Learning and Com-puting IPCSIT vol.3 ,IACSIT Press, Singapore., 1: 249-253.

[9] Diansheng Guo and Jeremy Mennis, 2009. Spatial data mining and geographic knowledge discovery-An introduction. Computers, Environment and Urban Systems., 33(6): 403-408.

[10] Dien J., K.M. Spencer and E. Donchin, 2005. Parsing the "Late Positive Complex": Mental chronometry and the ERP components that inhabit the neighborhood of the P300. Psychophysiology.

[11] Elnekave. S., M. Last and O. Maimon, 2007. Incremental Clustering of Mobile Objects. STDM07, IEEE. Engineering, Washington, DC, USA., 1: 422432.

[12] Gudmundsson, J. and M. Van Kreveld, 2006. Computing longest duration flocks in trajectory data. In ACM GIS., 35-42.

[13] Hoda M. O. Mokhtar et al., 2011. A Time Parameterized Technique for Clustering Moving Object Trajectories, International Journal of Data Mining \& Knowledge Management Process (IJDKP)., 1(1): 1430.

[14] Hoda Mokhtar and Jianwen Su, 2005. A query language for moving object trajectories. In SSDBM: Interna-tional Conference on Scientific and Statistical Data-base Management., 1: 173-182.

[15] Hoda, M. O. and Mokhtar, 2011. A Time Parameterized Technique for Clustering Moving Object Trajectories. International Journal of Data Mining \& Knowledge Management Process., 1(1): 1-17.

[16] Huang, Y., J. Pei and H. Xiong, 2006. Mining colocation patterns with rare events from spatial data sets. Geoinformatica., 10(3): 239-260. 
[17] Imam Mukhlash and Benhard Sitohang, 2007. Spatial Data Preprocessing for Mining Spatial Association Rule with Conventional Association Mining Algorithms. International Conference on Electrical Engineering and Informatics Institute Technology Bandung, Indonesia., 1: 531-534.

[18] Jensen. C,, D. Lin and B. Ooi, 2007. Continuous cluster-ing of moving objects. IEEE Trans. Knowl. Data Eng., 19(9): 1161-1174.

[19] Jiangping Chen, 2008. An Algorithm About Association Rule Mining Based On Spatial Autocorrelation. The International Archives of the Photogrammetry, Re-mote Sensing and Spatial Information Sciences. XXXVII( B6b), Beijing.

[20] Jiawei Han and Micheline Kamber, 2009. Data mining concepts and techniques. Morgan Kaufmann Publishers., 354-359.

[21] Jidong Chen et al., 2007. Clustering Moving Objects in Spatial Networks. Proceedings of the 12th international conference on Database systems for advanced applications., 1: 611-623.

[22] Kang Hye-Young., Joon-Seok and Ki-Joune, 2009. Simi-larity measures for trajectory of moving objects in cellular space. In SAC '09: Proceedings of the 2009 ACM symposium on Applied Computing., 1: 13251330.

[23] Kelejian, H.H. and I.R. Prucha, 2010. Specification and estimation of spatial autoregressive models with autoregressive and heteroskedastic disturbances. Jour-nal of Econometrics., 157(1): 53-67.

[24] Kuo. R.J., C.M. Chao and Y.T. Chiu, 2011. Application of particle swarm optimization to association rule mining. Applied Soft Computing., 11: 326-336.

[25] Lee Jae-Gil., Han Jiawei and Whang Kyu-Young, 2007. Trajectory clustering: a partition-andgroup frame-work. In SIGMOD '07: Proceedings of the 2007 ACM SIGMOD international conference on Management of data., 1: 593-604.

[26] Lin, X. and L.-F. Lee, L.-F, 2010. GMM estimation of spatial autoregressive models with unknown heteroskedasticity. Journal of Econometrics., 157 (1): 3452.

[27] Lu, Y. and J-C. Thill, 2008. Cross-scale analysis of clus-ter correspondence using different operational neigh-borhoods. Journal of Geographical Systems., 10(3): 241-261.

[28] Manisha Gupta, 2011. Application of Weighted Particle Swarm Optimization in Association Rule Mining. In-ternational Journal of Computer Science and Infor-matics (IJCSI) ISSN (PRINT): 2231 -5292., $1(3)$.

[29] Maragatham G et al, 2012. A Recent Review On Associ-ation Rule Mining. Indian Journal of Computer Science and Engineering (IJCSE)., 2(6): 831-836.
[30] Margaret H. Dunham., 2009. Data Mining Introductory and Advanced Topics. Dorling Kindersley (India) Pvt. Ltd., 1(1): 1-311.

[31] Richard Frank., Wen Jin and Martin Ester, 2009. Efficiently Mining Regional Outliers in Spatial Data., 1-18.

[32] Sigal Elnekave and Mark Last, 2007. A Compact Repre-sentation of Spatio-Temporal Data, Seventh IEEE International Conference on Data Mining Workshops.

[33] Sigal Elnekave et al., 2007. Predicting Future Locations Using Clusters' Centroids. Proceedings of the 15th annual ACM international symposium on Advances in geographic information systems., 55(1).

[34] Urška Demšar., Paul Harris., Chris Brunsdon., A. Stewart Fotheringham and Sean McLoone, 2012. Principal Component Analysis on Spatial Data: An Overview, Annals of the Association of American Geographers., 1:1-24.

[35] Veenu Mangat, 2010. Swarm Intelligence Based Technique for Rule Mining in the Medical Domain. International Journal of Computer Applications (0975 8887)., 4(1): 19-24.

[36] Yang, Z., C. Li and Y.K. Tse, 2006. Functional form and spatial dependence in dynamic panels. Economics Letters., 91: 138-145.

[37] Yiu, M.L. and N. Mamoulis, 2004. Clustering objects on a spatial network. In Proc. ACM SIGMOD.,1: 443454.

[38] Zhang Xuewu, 2008. Association Rule Mining on Spatio-temporalProcesses. Wireless Communications, Net-working and Mobile Computing, WiCOM '08., 1: 1- 4 . 